\title{
57. QUESTIONS CONCERNING THE USEFULNESS AND FEASIBILITY OF LARGE-SCALE STELLAR STATISTICS
}

\author{
W. SEITTER
}

Observatorium Hoher List der Universitäts-Sternwarte Bonn, Germany

\begin{abstract}
A proposal for a cooperative effort to secure three-color photometry of all stars up to the 18th magnitude of a $10^{\circ}$ field around the galactic equator has been made.
\end{abstract}

